\title{
The impact analysis of the gaps in kinematic pairs on dynamic processes in cyclic mechanisms
}

\author{
Viktor Telegin ${ }^{1, *}$ \\ ${ }^{1}$ Associate Professor, Lipetsk State Technical University, Lipetsk, Russia
}

\begin{abstract}
Cyclic mechanisms (cam, crank and others) are most vulnerable to the growth of dynamic (oscillatory) processes, especially at high speeds of their movement. Many factors affect the reliability and performance of these mechanisms, determined primarily by their dynamic characteristics. The main ones are the elastic-inertial and dissipative properties of the links, external forces acting on the elements of the mechanisms, as well as the gaps in the kinematic pairs of the links of the links. The paper studies impact of gaps in the kinematic pairs of link, design features of these links, all other things being equal, on the cyclic mechanical systems dynamics. Three computational models of kinematic pairs are considered in the article, the results of studying the influence these models parameters on the level of dynamic processes in the cam-lever mechanism during transfer workpieces between the cold-stamping automatic machine (CSAM) AB1818 positions.
\end{abstract}

\section{The studied mechanism and features of its work}

Figure 1 shows 3D model of the transfer mechanism cold-stamping automatic machine (CSAM) AB1818 [1]. In the process, the output link of the mechanism makes a reciprocating motion according to the scheme: forward motion - dwell - reverse motion dwell. The rotation speed of the cams is $300 \mathrm{rpm}$. The cam tracks are profiled according to the sinusoidal law of changing acceleration. When designing the mechanism, gaps in movable links are allowed: pusher - cam track (taking into account the clearance in the bearing of roller, cam manufacturing inaccuracies), gear transmission, crank mechanism of the transfer carriage drive. The cams and the transfer carriage are perfectly rigid body when performing research. The remaining elements of the mechanism are elastically deformable bodies.

The objective of the study is to determine the effect of the gap on the dynamics of the mechanism. In the framework of the studies presented in this paper, we assume that significant values of the gap will take place only in the link: pusher - cam track. The size of the remaining gaps is zero.

\footnotetext{
*Corresponding author: vv.telegin@yandex.ru
} 


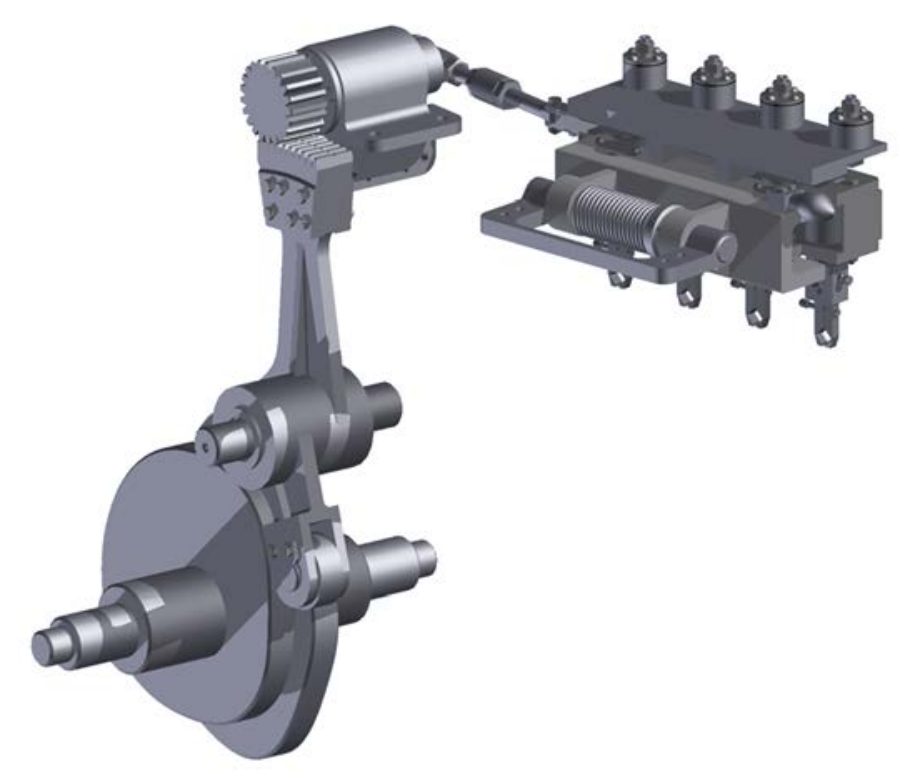

Fig. 1. 3D model of the transfer mechanism.

\section{The model of the mechanism and the software for calculating dynamic characteristics}

The calculation of oscillatory processes in the elements of mechanisms [2, 3] consists in representing them in the form of several masses sets into which the elastic-deformable links of mechanisms concentrated at points are divided. The masses are interconnected by inertialess, kinematic, piecewise continuous, dissipative links. As a result, the mathematical model of the mechanism is the several systems of nonlinear differential equations of the second order. The mass motion characteristics of the model can only be obtained numerically. The process of developing a model, its equations of motion, solving and analyzing the results obtained is rather laborious.

To automate the study of the dynamics of mechanisms, the author of the article developed software designed for dynamic analysis of mechanisms ("dam"). This solution is based on the methods of component modeling [4, 5], which allow constructing the initial calculation model from ready-made objects [6]. Figure 2 shows an object representation of the transfer mechanism in the "dam" software window.

Elastic-inertial parameters of the model: stiffness and mass (moments of inertia) are calculated on the basis of a solid-state model of the mechanism. The solid-state model itself is pre-built in one of the CAD systems [7]. The author used Autodesk Inventor Professional $[8,9,10]$ for this purpose. 


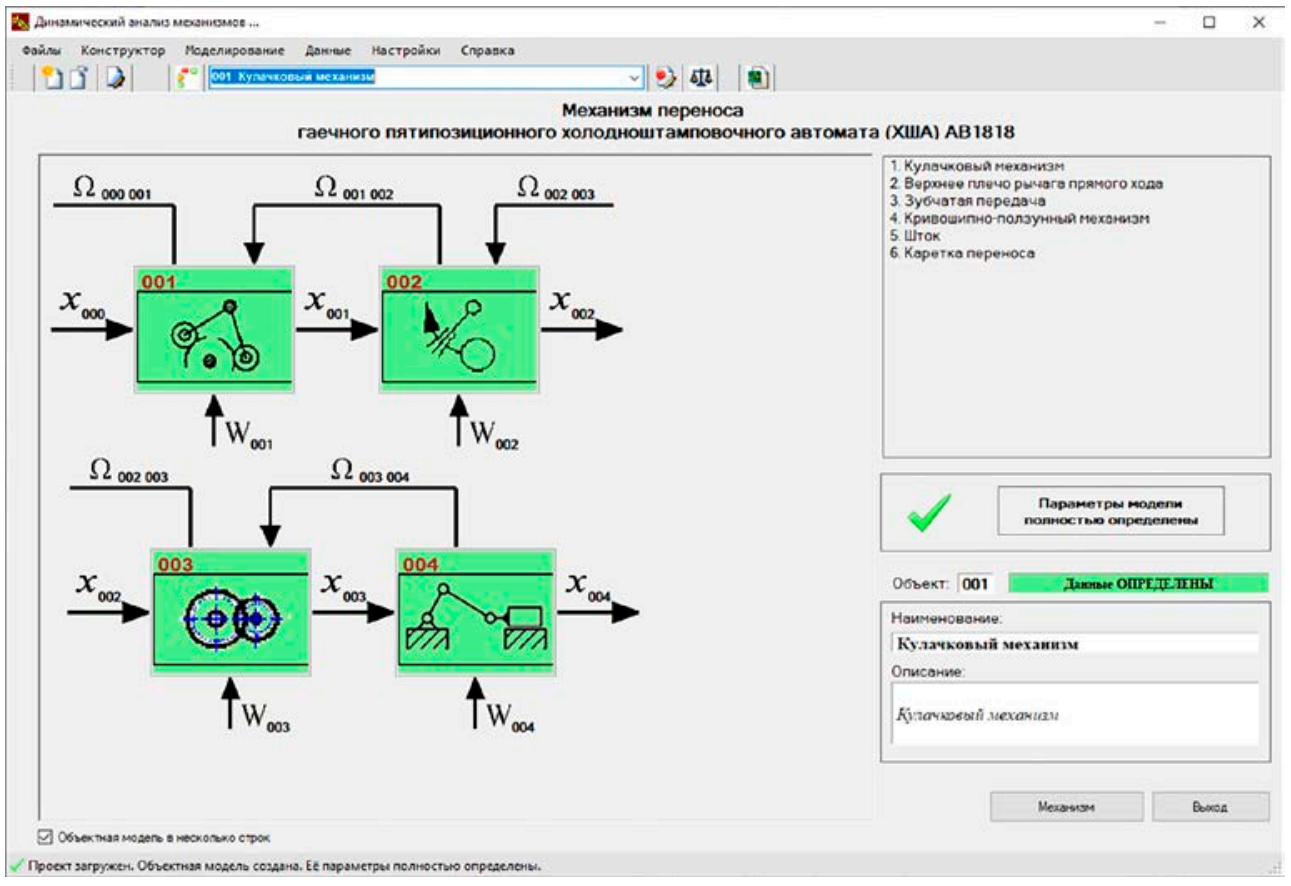

Fig. 2. Object representation of the mechanism.

\section{Computational models of mechanism links}

There are two categories of gaps: one-sided and two-sided (Figure 3). A one-sided gap can be considered as a special case of a two-sided gap, assuming that the stiffness of the connection two links under tension (or compression) is zero [11].

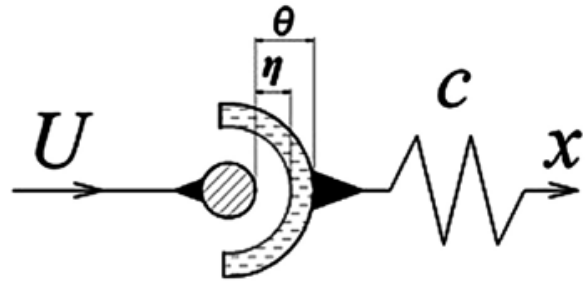

a

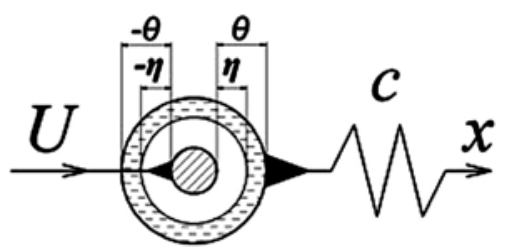

b

Fig. 3. Gap: $\boldsymbol{a}$ - one-sided, $\boldsymbol{b}$ - two-sided. $\boldsymbol{\theta}$ - gap considering contact deformation zone, $\boldsymbol{\eta}$ - onesided gap, $\boldsymbol{U}, \boldsymbol{x}$ - ideal and real link movement, $\boldsymbol{c}$ - link stiffness.

Consider the three most characteristic types of changes in the stiffness of the connection two links:

- The stiffness of the links when reconciling the gap changes almost instantly from zero to a certain final value (Figure 4a). This type of connect is defined by the term hard impact. The speed of an element instantly changes by a finite value, and the acceleration at this moment theoretically tends to infinity.

- After sampling the gap, the stiffness in the zone of contact deformation varies linearly (Figure 4b) - soft impact. Acceleration changes instantly by a finite amount. The speed change function is continuous. 
- After sampling the gap, the stiffness in the zone of contact deformation changes according to a sinusoidal law (Figure 4c). Impactless connection. The acceleration function is continuously.
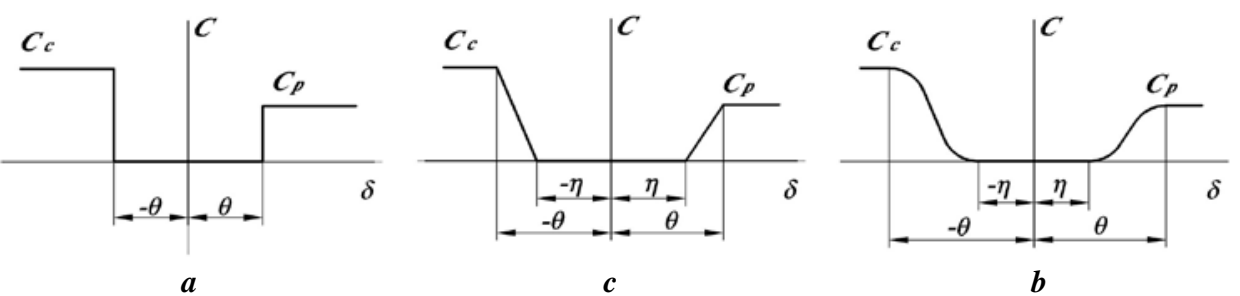

Fig. 4. Changing the stiffness value in the gaps: $\boldsymbol{a}-1 \mathrm{st}, \boldsymbol{b}-$ 2nd and $\boldsymbol{c}$-3rd types. $\boldsymbol{\theta}, \boldsymbol{\eta}$ - one-sided gap with and without considering contact deformation zone, $\boldsymbol{\delta}$ - deformation, $\boldsymbol{c}_{\boldsymbol{c}}, \boldsymbol{c}_{\boldsymbol{p}}$ - link stiffness under compression and tension.

The ratio of the gap zones (rupture of the kinematic chain) and contact deformation, when the stiffness begins to increase from zero to its maximum value, is determined by the ratio $\eta=\theta \cdot(1-k)$, where $\mathrm{k}$ is the coefficient of contact deformation. If $k=0$, then gap of the first type. If $k=1$, then there is no gap in the kinematic chain. The latter is characteristic of new mechanisms, the kinematic pairs of which have not yet "been worked out" and also of connections with gaps, the values of which are determined by the values of the tolerance field qualifications up to the 7 th -5 th.

We will consider the procedure for describing the process of connecting the links in conjunction with the gap using the example of a fragment of a dynamic model [11] (Figure 5).

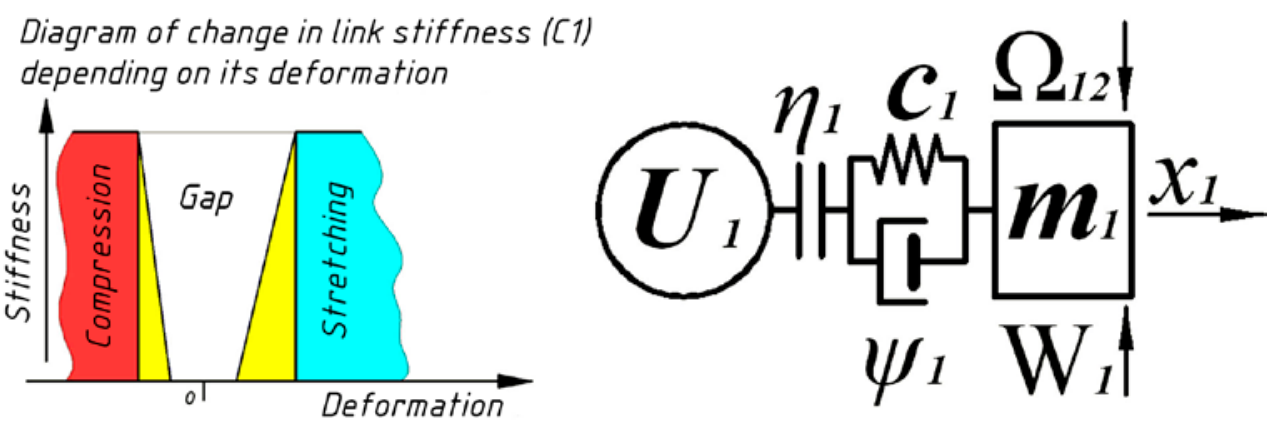

Fig .5. A fragment of a dynamic model typical object.

The equation of motion the mass $\mathrm{m} 1$ for this object model [1, 2]:

$$
m_{1} \ddot{x}_{1}=-c_{1} \cdot \delta-b_{1} \cdot\left(\dot{x}_{1}-\dot{U}_{1}\right)+W_{1}+\Omega_{12},
$$

where $m_{1}$ is mass, $c_{1}\left(c_{c}, c_{p}, \theta_{1}, \eta_{1}\right)$ is stiffness, $\delta=x_{1}-U_{1} \pm \eta_{1}, x_{1}$ is the actual displacement of the object, $b_{1}\left(\Psi_{1}, m_{1}, c_{1}\right)$ is the reduced dissipation coefficient, $U_{1}$ is the position function, $W_{1}$ - functions of external loads, $\Omega_{12}$ - response function.

The stiffness of the link, if the gap is open, is equal to zero $\left(\eta_{1} \geq 0, \eta_{1} \leq \theta\right)$ on the interval from $-\eta_{1}$ to $+\eta 1$. On the interval $\left|\theta_{1}-\eta_{1}\right|>0$, the stiffness increases from 0 to $c_{1}$ ( $c_{1}$ is equal to $c_{c}$ or $c_{p}$ ) instantly if the gap of 1 st type, according to a linear law or sinusoidal if the gap of 2nd or 3rd type respectively.

If $\theta_{1}>0,\left|\theta-\eta_{1}\right|>0, \eta_{1}=\theta_{1} \cdot(1-k)$, then there are the following calculated dependencies for the stiffness, deformation, and loads.

If the gap open, regardless of its type, then: $-\eta 1 \leq x 1-U 1 \leq+\eta 1, \delta=x 1-U 1, \quad c_{1}=0$. 
Table 1. The gap is selected. Compression.

\begin{tabular}{|c|c|c|}
\hline $\begin{array}{l}\text { Gap of the } \\
\text { 1st type }\end{array}$ & $\begin{array}{l}\text { Gap of the } \\
\text { 2nd type }\end{array}$ & $\begin{array}{l}\text { Gap of the } \\
\text { 3rd type }\end{array}$ \\
\hline $\begin{array}{l}x 1-U 1<-\eta 1, \\
\delta=x 1-U 1+\eta_{1}, \\
c_{1}=c_{c} .\end{array}$ & $\begin{array}{l}x 1-U 1<-\eta 1, \\
\delta=x 1-U 1+\eta_{1}, \\
a=\frac{c_{C}}{\eta 1-\theta_{1}}, \\
b=\frac{c_{c} \cdot \eta 1}{\eta 1-\theta_{1}}, \\
c_{1}=a \cdot(x 1-U 1)+b . \\
\text { If } c_{1} \geq c_{c}, c_{1}=c_{c} .\end{array}$ & $\begin{array}{l}x 1-U 1<-\eta 1, \\
\delta=x 1-U 1+\eta_{1}, \\
c_{1}=0.5 \cdot c_{C} \cdot \\
\cdot\left(\sin \left(\pi \cdot \frac{x 1-U 1-0.5 \cdot\left(\theta_{1}+\eta 1\right)}{\theta_{1}-\eta 1}\right)+1\right) . \\
\text { If } x 1-U 1 \leq-\theta_{1}, c_{1}=c_{c} .\end{array}$ \\
\hline
\end{tabular}

Table 2. The gap is selected. Tension.

\begin{tabular}{|c|c|c|}
\hline $\begin{array}{l}\text { Gap of the } \\
\text { 1st type }\end{array}$ & $\begin{array}{l}\text { Gap of the } \\
\text { 2nd type } \\
\end{array}$ & $\begin{array}{l}\text { Gap of the } \\
\text { 3rd type }\end{array}$ \\
\hline $\begin{array}{l}x 1-U 1>+\eta 1, \\
\delta=x 1-U 1-\eta_{1}, \\
c_{1}=c_{\mathrm{p}} .\end{array}$ & $\begin{array}{l}x 1-U 1>+\eta 1, \\
\delta=x 1-U 1-\eta_{1}, \\
a=-\frac{c_{\mathrm{p}}}{\eta 1-\theta_{1}}, \\
b=\frac{c_{p} \cdot \eta 1}{\eta 1-\theta_{1}}, \\
c_{1}=a \cdot(x 1-U 1)+b . \\
\text { If } c_{1} \geq c_{p}, c_{1}=c_{\mathrm{p}} .\end{array}$ & $\begin{array}{l}x 1-U 1>+\eta 1, \\
\delta=x 1-U 1-\eta_{1}, \\
c_{1}=0.5 \cdot c_{\mathrm{p}} \cdot \\
\cdot\left(\sin \left(\pi \cdot \frac{x 1-U 1-0.5 \cdot\left(\theta_{1}+\eta 1\right)}{\theta_{1}-\eta 1}\right)+1\right) . \\
\text { If } x 1-U 1>\theta_{1}, c_{1}=c_{p} .\end{array}$ \\
\hline
\end{tabular}

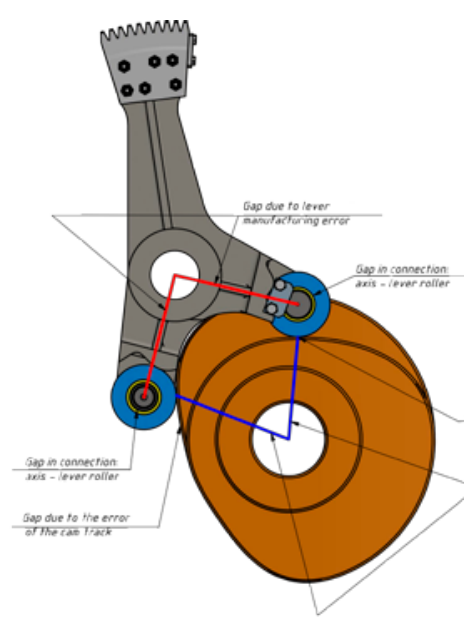

a

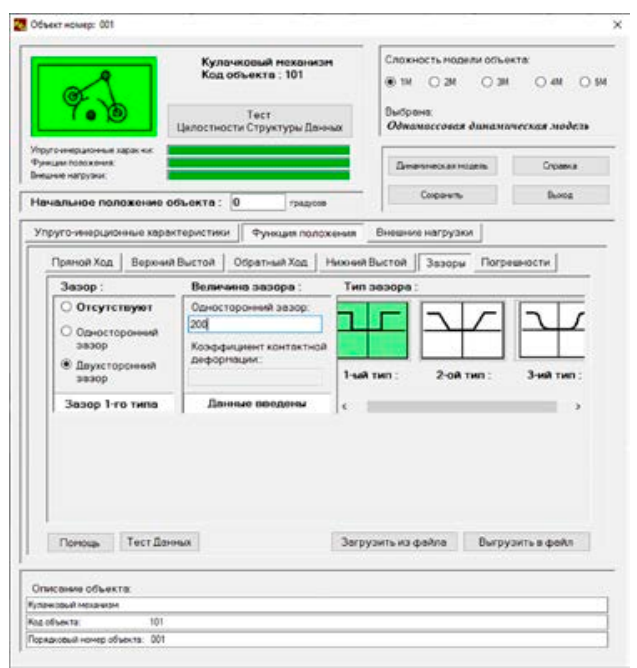

b

Fig. 6. $\boldsymbol{a}$ - gaps in the drive for transfer of the cam mechanism CSAM AB1818, $\boldsymbol{b}$ - determining the type and parameters of the gaps in the "dam" software. 
Taking approximately the fit of movable joints for a diameter of $50 \mathrm{H} 7 / \mathrm{h} 6$, we will have a minimum one-sided gap of the new mechanism for two-arm lever equal to $50 \mu \mathrm{m}$. In practice, its value can reach values up to $200 \mu \mathrm{m}$ due to tracks manufacturing errors of the cam block and the two-arm lever. This value is controlled during the assembly of the mechanism.

Figure 6 shows the position of the clearances in the cam mechanism [12] and shows a fragment of the "dam" program window in which the type and parameters of the gaps in the connection are set: axis of the lever roller - lever roller + lever roller - cam track.

\section{The study results of calculated parameters gaps to dynamics mechanical system}
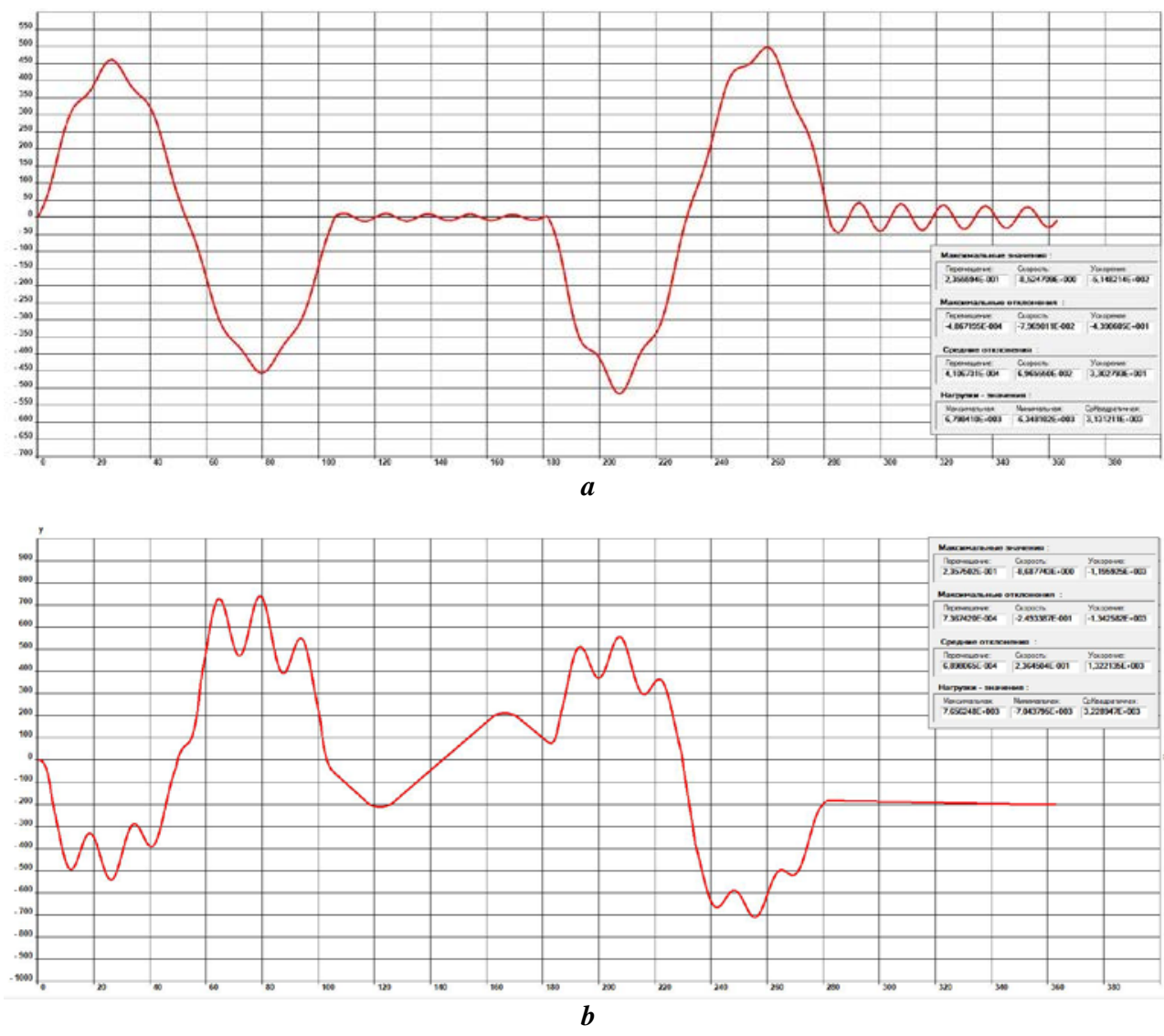

Fig. 7. Deviation the actual transfer carriage movement of the CSAM AB1818 from ideal in microns depending on the method for modeling gaps in its kinematic pairs: $\boldsymbol{a}$ - no gaps, $\boldsymbol{b}$-1st type of gaps. 

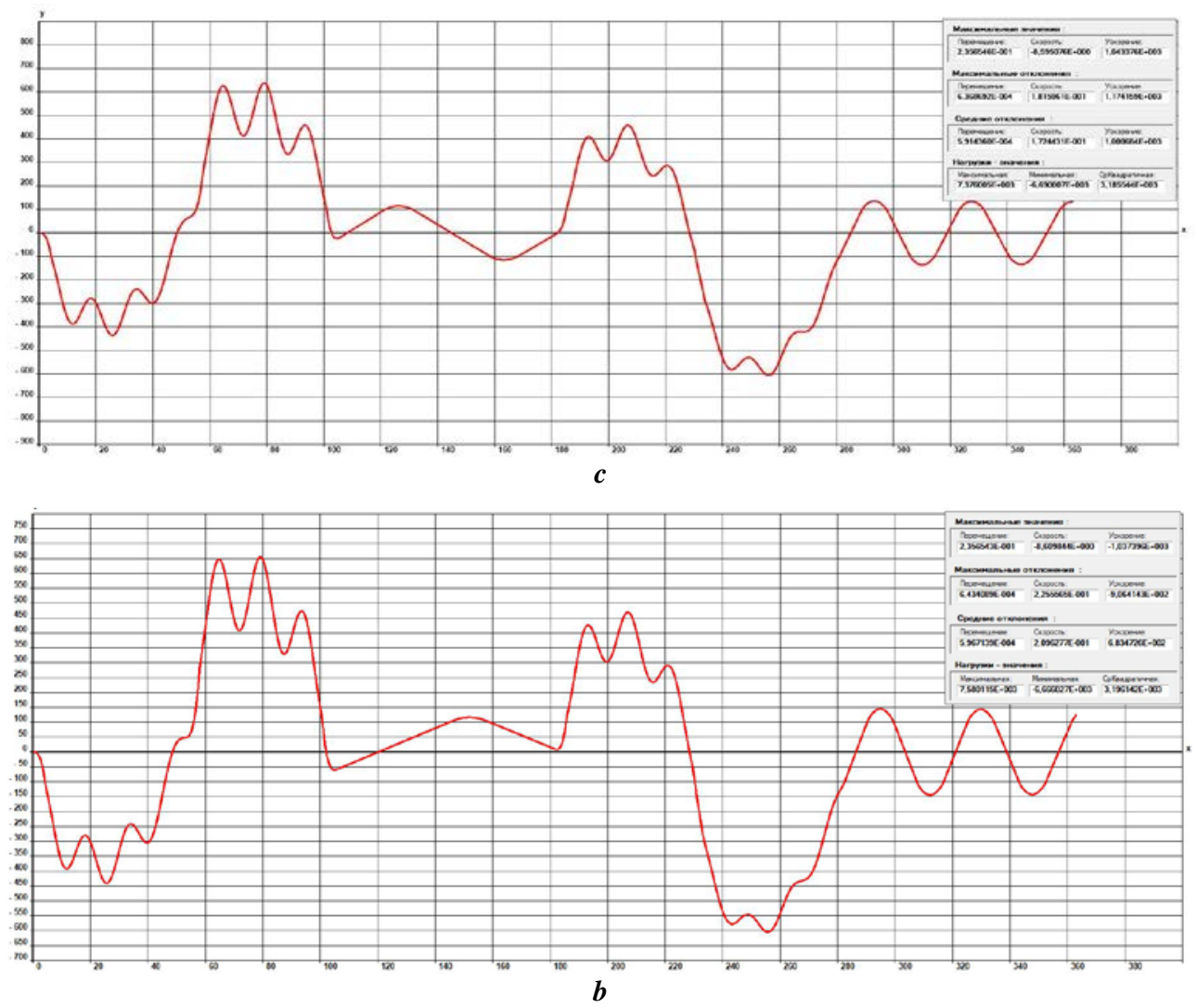

Fig. 8. Deviation the actual transfer carriage movement of the CSAM AB1818 from ideal in microns depending on the method for modeling gaps in its kinematic pairs: $\boldsymbol{a}$ - 2nd type, $\boldsymbol{b}$ - 3rd type of gaps.

As follows from the above dependences, the exclusion of gaps from the model of the crank-slide machine mechanism leads to a noticeable distortion of the calculation results (Figure 7a). Almost completely, there are no fluctuations in the output link of the cam mechanism in the sections of the dwellings. The other extreme is the use of the 1st type gaps in the model (Figure 7b). In this case, the gaps in the cam mechanism led to a noticeable deviation of the position of the output link and the exclusion from the calculation results of the oscillatory processes in the rear ditch section. The using gaps of the 2nd and 3rd type during modeling lead, in this case, to approximately the same results (Figures 8a and 8b). Additional studies conducted during the operation of mechanisms with higher speeds and in the presence of sharply changing technological forces have shown that the 3rd type gaps allow whiter correct modeling of dynamic processes.

\section{Conclusions}

Numerical experiments showed that with gaps corresponding to tolerances of less than the 5th-7th qualifications, as well as one-sided gaps in cam-pusher (or rocker) connections, the use of the 1st type gap model (Figure 4a) is quite justified, which leads to a reduction in the calculation volume. The use of gaps of the 2nd type (Figure 4b) can be recommended when modeling mechanisms in the absence of significant technological forces. 3rd type gaps (Figure 4c) allow the most accurate calculation of dynamic processes in the mechanisms of cyclic action. 


\section{References}

1. Y.A. Miropolsky Cold Die Forging on Automatic Machines (Moscow: Mashinostroine, 2001)

2. I.I. Wulfson, Vibrations in machines with cyclic action mechanisms (Leningrad: Mashinostroine, 1990)

3. V.L. Veits, M.Z. Kolovsky, A.E. Kochura, Dynamics of controlled machine aggregates (Moscow: Science, 1984)

4. E.S. Benkovsky, Yu. B. Kolesov, Yu. B. Senichenkov, Dynamic System Practical Modeling (St. Petersburg: BHV-Petersburg, 2002)

5. J. Rumbaugh et al, Object-Oriented Modeling and Design (Prentice Hall, 1991)

6. V. Telegin, A. Kozlov, Computer realization of research into the dynamics of mechanical systems, IOP Conference Series: Materials Science and Engineering, v. 124, pp. 012101 (2016)

7. V. Bolshakov, A. Bochkov, Yu. Lyachek, Solid Modeling of Parts in CAD-systems: AytoCAD, Compass -3D, SolidWorks, Inventor, Creo (S-Pb.: Peter, 2015)

8. V.V. Telegin, I.V. Telegin, A.V. Kirichek, Solid-state modeling and basic training of specialists in the field of mechanical engineering, IOP Conf. Series: Materials Science and Engineering v. 483, pp. 012004 (2019)

9. V.V. Telegin, A.M. Kozlov, A.V. Kirichek, Solid Modeling in Autodesk Inventor at Initial Stage of Training of Specialists in Field Mechanical Engineering, Lecture Notes In Mechanical Engineering ISSN: 2195-4356, v. Part F4, pp. 1241-1247 (2019)

10. V.V. Telegin, A.M. Kozlov, V.I. Sakalo, Solid Modeling and Dynamic Analysis of Mechanisms of Press-forging Machines, Procedia Engineering, v. 206, pp. 1258-1263 (2017)

11. V. Telegin, A. Kozlov, T. Shumilova, Development and research of the rotating lever object as a dynamic model of a cycle mechanism, MATEC Web Conf. International Conference on Modern Trends in Manufacturing Technologies and Equipment (ICMTMTE 2018), v. 224, pp. 02078 (2018)

12. V.F. Utkin, Yu.V. Kryuchkov, Reliability and efficiency in engineering: Reference Book (Vol. 3) (Moscow: Efficiency of Engineering Systems, 1988) 